PROCEEDINGS OF THE

AMERICAN MATHEMATICAL SOCIETY

Volume 127, Number 1, January 1999, Pages 279-288

S 0002-9939(99)04482-2

\title{
THE ASSOCIATED VARIETY OF AN INDUCED REPRESENTATION
}

\author{
DAN BARBASCH AND MLADEN BOŽIČEVIĆ
}

(Communicated by Roe Goodman)

\begin{abstract}
This paper studies the behavior of the associated variety under induction from real parabolic subgroups. We derive a formula for the associated variety of an induced module which is analogous to the formula for the wave front set of a derived functor module obtained by Barbasch and Vogan.
\end{abstract}

\section{INTRODUCTION}

The idea of studying a representation of a real Lie group by attaching to it an invariant which is a union of nilpotent orbits has a long history and has proved very fruitful. In particular in the case of a reductive group, there are the analytic notions of asymptotic support $A S(\pi)$ and wave front set $W F(\pi)$ introduced by Howe and Barbasch-Vogan (they in fact coincide). Roughly they measure the degree to which the distribution character of the representation is singular. Generalizing a notion coming from primitive ideals, there is the algebraic notion of associated variety $A V(\pi)$ introduced by Vogan. Roughly this measures the size of the annihilator of a single vector in the representation.

The analytic notions behave well under induction from real parabolic subgroups. We use the notation in section 1 . If $\mathfrak{p}=\mathfrak{m}+\mathfrak{u}$ is the complexification of a real parabolic subalgebra, let $\pi$ be an $(\mathfrak{m}, M \cap K)$-module. Then $W F\left(\operatorname{Ind}_{P_{\mathbb{R}}}^{G_{\mathbb{R}}}[\pi]\right)$ is the union of the nilpotent orbits which meet $W F(\pi)+\mathfrak{u}_{\mathbb{R}}$ in an open set.

Similarly, the algebraic notion behaves well under derived functor induction from $\theta$-stable parabolic subalgebras. If $\mathfrak{q}=\mathfrak{l}+\mathfrak{u}$ is such a parabolic subalgebra and $\pi$ is an $(\mathfrak{l}, L \cap K)$-module, then $A V\left(\mathcal{R}_{\mathfrak{q}}^{s}[\pi]\right)$ is the union of closures of nilpotent $K$-orbits in $\mathfrak{s}$ which meet $A V(\pi)+(u \cap \mathfrak{s})$ in an open set.

For the case of the WF-set of a derived functor module, a computation can be found in $[\mathrm{BV}]$. Roughly the result is as follows:

$$
W F\left(\mathcal{R}_{\mathfrak{q}}^{s}[\pi]\right)=C l\left[\bigcup_{t>0} A d G_{\mathbb{R}}[t \rho(\mathfrak{u})+W F(\pi)]\right] \backslash \bigcup_{t>0} A d G_{\mathbb{R}}[t \rho(\mathfrak{u})+W F(\pi)] .
$$

(The result is proved for $\pi$ one-dimensional, but the proof very likely carries over to the more general case.) The purpose of this note is to show that a similar result (Theorem 3.4) holds for $A V(\pi)$ and real induction. This result follows in a more or less straightforward manner from the formulas for the characteristic variety in [Gi] combined with some results in $[\mathrm{SW}]$.

Received by the editors October 20, 1996 and, in revised form, April 30, 1997.

1991 Mathematics Subject Classification. Primary 22E46.

(C)1999 American Mathematical Society 
We envision that this would be useful for computing with the associated variety of an induced unipotent representation (in the real case these are not closures of single nilpotent orbits, e.g. [B]). This allows one to deal with both types of induction exclusively in the algebraic setting.

The relation between $W F(\pi)$ and $A V(\pi)$ has been the subject of intense study in recent years in the work of J.T. Chang, Rossmann and culminating with the work of Schmid and Vilonen. These results are quite powerful; very likely this result can be derived from their work. However it seems to us that the formula in Theorem 3.4 is fairly elementary and deserves a short self-contained treatment.

It would be even nicer to have a proof of 3.4 without recourse to characteristic varieties of $\mathrm{D}$-modules. We do not know such a proof. For example, formula (0.1) could be proved by using the (much harder) analogue of the formula in [Gi] for $\mathbb{R}$-constructible sheaves ([SV]), by essentially the same method employed in this paper. In contrast, the proof of $(0.1)$ in $[\mathrm{BV}]$ only uses standard results about characters.

\section{Preliminaries And notation}

In this section we fix the notation and recall the main result from [Ch]. In order to simplify the exposition our assumptions on the group will be slightly restrictive. However the main result of this note can be extended to the Lie groups of HarishChandra class.

Let $G_{\mathbb{R}}$ be a real semisimple Lie group. In addition we assume that $G_{\mathbb{R}}$ is connected and linear. We fix a maximal compact subgroup $K_{\mathbb{R}}$ of $G_{\mathbb{R}}$ and write $G$ and $K$ for the complexifications of $G_{\mathbb{R}}$ and $K_{\mathbb{R}}$. We denote further by $\mathfrak{g}_{\mathbb{R}}$ and $\mathfrak{g}$ the Lie algebras of $G_{\mathbb{R}}$ and $G$. Let $\theta$ be the Cartan involution on $\mathfrak{g}_{\mathbb{R}}$ determined by the choice of $K_{\mathbb{R}}$. We denote by the same letter the corresponding involution on $\mathfrak{g}$. We write

$$
\mathfrak{g}_{\mathbb{R}}=\mathfrak{k}_{\mathbb{R}}+\mathfrak{s}_{\mathbb{R}}, \quad \text { resp. } \quad \mathfrak{g}=\mathfrak{k}+\mathfrak{s},
$$

for the eigenspace decomposition of $\theta$. Given a Cartan subalgebra $\mathfrak{h}$ of $\mathfrak{g}$ and an $\mathfrak{h}$-invariant subspace $\mathfrak{m}$, we will denote by $\Delta(\mathfrak{m})$ the set of nonzero $\mathfrak{h}$-weights on $\mathfrak{m}$ and by $2 \rho(\mathfrak{m})$ the sum of weights from $\Delta(\mathfrak{m})$.

Let $X$ be the flag variety of Borel subalgebras of $\mathfrak{g}$. Recall that the group $K$ acts on $X$ with finitely many orbits. Next we will construct a family of $K$-invariant open sets in $X$ that are closely related to induced modules.

First we choose a maximally split $\theta$-stable Cartan subalgebra $\mathfrak{h}_{\mathbb{R}}$ in $\mathfrak{g}_{\mathbb{R}}$. We denote by $\mathfrak{h}$ the complexification of $\mathfrak{h}_{\mathbb{R}}$ and fix a Borel subalgebra $\mathfrak{b}$ containing $\mathfrak{h}$ and having the property that the corresponding positive root system is $(-\theta)$-stable outside the imaginary roots. Then all the imaginary roots are necessarily compact. We denote by $x_{0}$ the point in $X$ representing $\mathfrak{b}$.

Let $h_{0} \in \mathfrak{h}_{\mathbb{R}}$. Denote by $\mathfrak{p}$ the parabolic subalgebra of $\mathfrak{g}$ defined by $h_{0}$. In more detail, we can write a Levi decomposition of $\mathfrak{p}$ as

$$
\mathfrak{p}=\mathfrak{l}+\mathfrak{u},
$$

where $\mathfrak{l}$ is the centralizer of $h_{0}$ in $\mathfrak{g}$ and

$$
\Delta(\mathfrak{u})=\left\{\alpha \in \Delta(\mathfrak{g}): \alpha\left(h_{0}\right)>0\right\} .
$$

Furthermore we write

$$
\mathfrak{l}=\mathfrak{m}+\mathfrak{z},
$$


with $\mathfrak{m}$ the semisimple part and $\mathfrak{z}$ the center of $\mathfrak{l}$. Let $P$ be the normalizer of $\mathfrak{p}$ in $G$. We consider the Levi decomposition of $P$,

$$
P=L U,
$$

compatible with the decomposition for the Lie algebras. We write

$$
L=M Z \text {, }
$$

where $M$ is the derived group of $L$ and $Z$ the identity component of the center. Let $Y$ be the generalized flag variety of parabolic subalgebras conjugate to $\mathfrak{p}$. Let

$$
p: X \longrightarrow Y
$$

be the natural projection. Denote by $y_{0}$ the point in $Y$ corresponding to $\mathfrak{p}$, and put $X_{0}=p^{-1}\left(y_{0}\right)$.

1.1 Lemma. Let $y \in Y$ and let $\mathfrak{p}_{y}$ be the corresponding parabolic subalgebra. Choose a $\theta$-stable Cartan subalgebra $\mathfrak{h}_{y}$ and consider a Levi decomposition

$$
\mathfrak{p}_{y}=\mathfrak{l}_{y}+\mathfrak{u}_{y} .
$$

Then the orbit K.y is open in $Y$ if and only if $\Delta\left(\mathfrak{u}_{y}\right)$ is $(-\theta)$-stable. In particular, the orbit K. $y_{0}$ is open in $Y$.

Proof. By considering the tangent space to $K . y$ at $y$, we conclude that $K . y$ is open in $Y$ if and only if $\operatorname{dim}\left(\mathfrak{u}_{y}\right)=\operatorname{dim}\left(\mathfrak{k} / \mathfrak{k} \cap \mathfrak{p}_{y}\right)$. For any root $\alpha \in \Delta$ we choose a nonzero root vector $X_{\alpha} \in \mathfrak{g}_{\alpha}$. Suppose that the condition of the lemma is true. It will suffice to check that the vectors $X_{-\alpha}+\theta X_{-\alpha}, \alpha \in \Delta\left(\mathfrak{u}_{y}\right)$, are linearly independent $\bmod \mathfrak{k} \cap \mathfrak{p}_{y}$. But this is clear, since otherwise the vectors $X_{-\alpha}, \alpha \in \Delta\left(\mathfrak{u}_{y}\right)$, would be linearly dependent $\bmod \mathfrak{p}_{y}$, which is not possible. Conversely, by the definition of $\mathfrak{p}$ we obtain immediately that $\theta \Delta(\mathfrak{u})=-\Delta(\mathfrak{u})$, and therefore $K y_{0}$ is open in $Y$. Suppose that $K . y$ is open in $Y$. Then $y \in K . y_{0}$, and this implies that $\theta \Delta\left(\mathfrak{u}_{y}\right)=$ $-\Delta\left(\mathfrak{u}_{y}\right)$.

For a smooth algebraic variety $X$ we denote by $\mathcal{D}_{X}$ the sheaf of differential operators with algebraic coefficients. Let $f: X \longrightarrow Y$ be a map of algebraic varieties and let $\mathcal{D}$ be a twisted sheaf of differential operators on $Y$ (tdo in short). Then through $f$ we have a tdo $\mathcal{D}^{f}$ on $X$, and we denote by $f^{+}$the inverse image functor from the category of $\mathcal{D}$-modules to the category of $\mathcal{D}^{f}$-modules and by $f_{+}$ the direct image functor from the category of $\mathcal{D}^{f}$-modules to the category of $\mathcal{D}$ modules. Let $A$ be an algebraic group acting on $X$. Given a tdo $\mathcal{D}$ on $X$ endowed with an $A$-action, we will denote by $\mathcal{M}(\mathcal{D}, A)$ the category of $\mathcal{D}$-modules with a compatible action of $A$. Finally, given a $\mathcal{D}$-module $\mathcal{F}$, we denote by $\Gamma(X, \mathcal{F})$ the group of global sections of $\mathcal{F}$.

Having introduced the requisite notation, we now explain one consequence of the duality theorem from $[\mathrm{Ch}]$ (compare also $[\mathrm{HMSW}]$ ). First we return to the setting before Lemma 1. Recall that for $\lambda \in \mathfrak{h}^{*}$ there are tdos $\mathcal{D}_{X, \lambda}$ on $X$ and $\mathcal{D}_{X_{0}, \lambda}$ on $X_{0}$. We assume that the parameter $\lambda$ is antidominant with respect to $\mathfrak{g}$. Let $\mathcal{V}$ be an object from $\mathcal{M}\left(\mathcal{D}_{X_{0}, \lambda}, K \cap L_{0}\right)$. Let $Q$ be the $K$-orbit of $\mathfrak{p}$. We set $p^{-1}(Q)=Z$ and denote by

$$
i: X_{0} \longrightarrow Z \text { and } j: Z \longrightarrow X
$$

the natural inclusions. Then the restriction functor

$$
i^{+}: \mathcal{M}\left(\mathcal{D}_{Z, \lambda}, K\right) \longrightarrow \mathcal{M}\left(\mathcal{D}_{X_{0}, \lambda}, K \cap L_{0}\right)
$$


is an equivalence of categories. Therefore we can find $\mathcal{W}$ from $\mathcal{M}\left(\mathcal{D}_{Z, \lambda}, K\right)$, whose restriction to $X_{0}$ is $\mathcal{V}$. We recall that for an $\mathcal{M}\left(\mathcal{D}_{X, \lambda}, K\right)$-module $\mathcal{F}$, the global sections $\Gamma(X, \mathcal{F})$ have a natural structure of $(\mathfrak{g}, K)$-module. As a last ingredient we denote by $\operatorname{Ind}_{\bar{P}_{\mathbb{R}}}^{G_{\mathbb{R}}}$ the normalized parabolic induction (i.e. the usual shift is taken into account) which maps (ll, $K \cap L)$-modules to ( $\mathfrak{g}, K)$-modules ([Vo], 4.1). Here $\bar{P}$ denotes the parabolic opposite to $P$. The following result is then an immediate consequence of the duality theorem:

1.2 Proposition. $\Gamma\left(X, j_{+} \mathcal{W}\right) \simeq \operatorname{Ind}_{\bar{P}_{\mathbb{R}}}^{G_{\mathbb{R}}}\left(V \otimes \mathbb{C}_{\rho(\mathfrak{u})}\right)$ as $(\mathfrak{g}, K)$-modules.

\section{Some Results about CHaRACTERISTIC VARIETIES}

We begin with some general considerations, some of which can also be found in $[\mathrm{ABV}]$. Let $X$ be a smooth complex algebraic variety. We write $T^{*} X$ for the cotangent bundle of $X$. Let $f: X \longrightarrow Y$ be a map between smooth complex algebraic varieties. Then we write $\rho_{f}: X \times_{Y} T^{*} Y \longrightarrow T^{*} X$ for the differential of $f$, and $\omega_{f}: X \times_{Y} T^{*} Y \longrightarrow T^{*} Y$ for the projection. In particular, if $M$ is a smooth subvariety of $X$ and $i: M \longrightarrow X$ the corresponding embedding, then the conormal bundle to $M$ is defined by $T_{M}^{*} X=\omega_{i}\left(\operatorname{ker} \rho_{i}\right)$. We denote by $C h(\mathcal{V})$ the characteristic variety of a $\mathcal{D}_{Y}-$ module $\mathcal{V}$. If $f$ is non-characteristic for $\mathcal{V}$, i.e. if

$$
\operatorname{ker} \rho_{f} \cap \omega_{f}^{-1}(C h(\mathcal{V})) \subset X \times_{Y} T_{Y}^{*} Y,
$$

then the characteristic variety of the inverse image $C h\left(f^{+}(\mathcal{V})\right)$ is related to $C h(\mathcal{V})$ by the formula $[\mathrm{Ka}]$ :

$$
C h\left(f^{+}(\mathcal{V})\right)=\rho_{f} \omega_{f}^{-1}(C h(\mathcal{V})) .
$$

Next we will study the behavior of characteristic varieties of equivariant $\mathcal{D}$-modules under induction. Let $G$ be a complex algebraic group and $A$ an algebraic subgroup. Suppose that $A$ acts on a variety $X$. Then the associated bundle $G \times{ }^{A} X$ is defined as the qoutient of $G \times X$ by a free $A$-action

$$
a .(g, x)=\left(g a^{-1}, a x\right), \quad a \in A, g \in G, x \in X .
$$

We write $[g, x]$ for the class corresponding to $(g, x) \in G \times X$. We assume now that $A$ acts on $X$ with finitely many orbits. Let $\mathcal{V}$ be a $\left(\mathcal{D}_{X}, A\right)$-module. Then we can find $A$-orbits $Q_{1}^{\prime}, \ldots ., Q_{r}^{\prime}$ such that

$$
C h(\mathcal{V})=T_{Q_{1}^{\prime}}^{*} X \cup \ldots \cup T_{Q_{r}^{\prime}}^{*} X .
$$

Set $Y=G \times{ }^{A} X$ and let $i: X \longrightarrow Y, i(x)=[e, x](e$ is the identity element in $G)$ be the natural embedding. Then the map

$$
i^{+}: \mathcal{M}\left(\mathcal{D}_{Y}, G\right) \longrightarrow \mathcal{M}\left(\mathcal{D}_{X}, A\right)
$$

is an equivalence of categories. Let $\mathcal{V}$ be a $\mathcal{M}\left(\mathcal{D}_{Y}, A\right)$-module.

2.1 Proposition. With the above notation let $\mathcal{W}$ be a $\left(\mathcal{D}_{Y}, G\right)$-module such that $i^{+} \mathcal{W}=\mathcal{V}$. Then $\rho_{i}$ is injective on $\omega_{i}^{-1}(C h(\mathcal{W}))$, and we have

$$
C h(\mathcal{W})=T_{Q_{1}}^{*} Y \cup \ldots \cup T_{Q_{r}}^{*} Y,
$$

where $Q_{i}=G \cdot Q_{i}^{\prime}$. 
Proof. We identify $X$ with its image in $Y$. Then any $G$-orbit $Q$ on $Y$ can be written as $Q=G . x, x \in X$. In order to verify that $\mathcal{W}$ is non-characteristic for $i$ it will suffice to check the following transversality condition:

$$
T_{x} Q+T_{x} X=T_{x} Y .
$$

The map $G \times X \longrightarrow Y,(g, x) \mapsto[g, x]$ is a fibration with fibres isomorphic to $A$. This fact implies the above transversality condition, since $\{e\} \times X$ and $G \times\{x\}$ are transversal in $G \times X$. Let $Q^{\prime}=Q \cap X$. Then as a consequence of the transversality and the fact that the stabilizers of $x$ in $G$ and $A$ are equal we obtain

$$
T_{x} Y / T_{x} Q \simeq T_{x} X / T_{x} Q^{\prime} .
$$

This implies the second statement.

Next we explain some notation related to homogeneous vector bundles. Let $G$ and $A$ be as above. For a subspace $V \subset \mathfrak{g}$ we will denote by $V^{\perp}$ the subspace of linear forms on $\mathfrak{g}$ vanishing on $V$. We assume that $V^{\perp}$ is stable for the coadjoint action $A d$ of $A$ on $\mathfrak{g}^{*}$. Then we have the associated bundle $E=G \times{ }^{A} V^{\perp}$. In particular, the vector bundle $G \times{ }^{A} \mathfrak{g}^{*}$ is trivial, and the isomorphism $\alpha_{G / A}: G \times{ }^{A}$ $\mathfrak{g}^{*} \simeq G / A \times \mathfrak{g}^{*}$ is given by

$$
\alpha_{G / A}([g, \xi])=(g A, A d(g) \xi), g \in G, \xi \in \mathfrak{g}^{*} .
$$

Let $V_{1} \subset V$ be an $A$-stable subspace. Then we have a natural embedding $G \times{ }^{A}$ $V^{\perp} \hookrightarrow G \times{ }^{A} V_{1}^{\perp}$ of homogeneous vector bundles over $G / A$. In particular, by composing the embedding $E \hookrightarrow G \times{ }^{A} \mathfrak{g}^{*}$ and $\alpha_{G / A}$ we obtain an embedding of $E$ into the trivial bundle $G / A \times \mathfrak{g}^{*}$. Whenever we write $(g A, \nu)$ for an element in $E$, we are considering $E$ as a subbundle of $G / A \times \mathfrak{g}^{*}$.

We return to the setting of the flag variety $X$ introduced in section 1 . In particular we have a Borel subalgebra $\mathfrak{b}$ of $\mathfrak{g}$, and we denote by $B$ its normalizer in $G$. Write

$$
B=H N
$$

for a Levi decomposition of $B$, where $H$ is a Cartan subgroup and $N$ the unipotent radical. Set

$$
\tilde{X}=G / N
$$

and denote by $\tilde{x}_{0}$ the identity coset. We will consider the left $G \times H$-action on $\tilde{X}$ defined by the formula

$$
(g, h) \cdot g^{\prime} \tilde{x}_{0}=g g^{\prime} h^{-1} \tilde{x}_{0} \quad g, g^{\prime} \in G, h \in H .
$$

Then the $H$-action is free and the natural projection

$$
\pi: \tilde{X} \longrightarrow X
$$

can be viewed as the quotient map. Given a tdo $\mathcal{D}_{X, \lambda}$ on $\tilde{X}$, we have a tdo $\mathcal{D}_{X, \lambda}^{\pi}$ on $X$. By the result A.1.3 from [HMSW] we actually have $\mathcal{D}_{\tilde{X}} \simeq \mathcal{D}_{X, \lambda}^{\pi}$. Hence, if $\mathcal{V}$ is a $\mathcal{D}_{X, \lambda}$-module, then $\pi^{+}(\mathcal{V})$ is a $\left(\mathcal{D}_{\tilde{X}}, H\right)$-module. Furthermore, since $\pi$ is smooth, we can apply the formula for the non-characteristic inverse image to compute $C h\left(\pi^{+} \mathcal{V}\right)$. 
Clearly the cotangent bundle $T^{*} \tilde{X}\left(T^{*} X\right)$ is naturally isomorphic to $G \times^{N} \mathfrak{n}^{\perp}$ $\left(G \times \times^{B} \mathfrak{b}^{\perp}\right)$. It is not difficult to show that under this isomorphism, the $H$-action on $T^{*} \tilde{X}$ which is induced by the $H$-action on $\tilde{X}$ is given by

$$
h .[g, \xi]=\left[g h^{-1}, A d(h) \xi\right], \quad g \in G, h \in H, \xi \in \mathfrak{n}^{\perp} .
$$

Now we define a vector bundle $\tilde{\mathfrak{g}}^{*}$ over $X$ by setting $\tilde{\mathfrak{g}}^{*}=G \times{ }^{B} \mathfrak{n}^{\perp}$. Since $T^{*} X=$ $G \times{ }^{B} \mathfrak{b}^{\perp}$, we may consider $T^{*} X$ as a subbundle of $\tilde{\mathfrak{g}}^{*}$. Let

$$
\beta: T^{*} \tilde{X} \longrightarrow \tilde{\mathfrak{g}}^{*}
$$

be the quotient map for the $H$-action on $T^{*} \tilde{X}$. If we realize $T^{*} \tilde{X}\left(\tilde{\mathfrak{g}}^{*}\right)$ as a subbundle of $\tilde{X} \times \mathfrak{g}^{*}\left(X \times \mathfrak{g}^{*}\right)$ via $\alpha_{G / N}\left(\alpha_{G / B}\right)$, then the map $\beta$ is given by $\beta(\tilde{x}, \nu)=(\pi(\tilde{x}), \nu), \tilde{x} \in \tilde{X}, \nu \in \mathfrak{g}^{*}$. Further we denote by $\gamma$ the map

$$
\gamma: \tilde{\mathfrak{g}}^{*} \longrightarrow \mathfrak{g}^{*}
$$

obtained by composing the embedding $\alpha_{G / B}$ and the projection $X \times \mathfrak{g}^{*} \longrightarrow \mathfrak{g}^{*}$. Clearly $\gamma\left(T^{*} X\right) \subset \mathcal{N}^{*}$, where $\mathcal{N}^{*}$ denotes the nilpotent cone in $\mathfrak{g}^{*}$.

2.2 Lemma. Let $\mathcal{F}$ be a $\mathcal{D}_{X, \lambda}-$ module. Then $\beta\left(C h\left(\pi^{+} \mathcal{F}\right)\right)=C h(\mathcal{F})$, and as a consequence we obtain $\gamma\left(\beta\left(C h\left(\pi^{+} \mathcal{F}\right)\right)\right)=\gamma(C h(\mathcal{F}))$.

Proof. Let $\left(\tilde{x},\left(x, \xi_{x}\right)\right) \in \omega_{\pi}^{-1}(C h(\mathcal{F}))$. Then we have

$$
\beta \rho_{\pi}\left(\tilde{x},\left(x, \xi_{x}\right)\right)=\beta\left(\tilde{x}, \xi_{x}\right)=\left(x, \xi_{x}\right) .
$$

The claim follows.

Next we will apply 2.1 in a specific case. We draw attention to the notation introduced before 1.2. Furthermore, we set

$$
\tilde{Z}=\pi^{-1}(Z), \quad \tilde{j}: \tilde{Z} \longrightarrow \tilde{X}, \quad \tilde{X}_{0}=\pi^{-1}\left(X_{0}\right), \quad \tilde{i}: \tilde{X}_{0} \longrightarrow \tilde{Z} .
$$

We will denote the restriction of $\pi$ to any subset of $\tilde{X}$ also by $\pi$. Then we want to relate the characteristic varieties of

$$
\tilde{\mathcal{W}}=\pi^{+} \mathcal{W} \text { and } \tilde{\mathcal{V}}=\pi^{+} \mathcal{V}
$$

Given $x \in X$, we denote by $\mathfrak{b}_{x}$ the corresponding Borel subalgebra and by $\mathfrak{n}_{x}$ its nilpotent radical. We fix a splitting $\mathfrak{g}=\mathfrak{l}+\mathfrak{u}+\theta \mathfrak{u}$ and use it to define the embedding $l: \mathfrak{l}^{*} \longrightarrow \mathfrak{g}^{*}$. We denote also by $l$ the following embeddings:

$$
\tilde{X}_{0} \times \mathfrak{l}^{*} \longrightarrow \tilde{X} \times \mathfrak{g}^{*}, \quad X_{0} \times \mathfrak{l}^{*} \longrightarrow X \times \mathfrak{g}^{*} .
$$

2.3 Lemma. The map $l$ has the following properties:

(1) $l\left(T^{*} \tilde{X}_{0}\right) \subset T^{*} \tilde{X}$

(2) $l\left(\tilde{\mathfrak{I}}^{*}\right) \subset \tilde{\mathfrak{g}}$

(3) $l\left(T^{*} X_{0}\right) \subset T^{*} X$.

Proof. We will verify only the first statement. Let $x \in X_{0}$. Since $L$ normalizes $\mathfrak{u}$ we have

$$
\mathfrak{n}_{x}=\mathfrak{n}_{x} \cap \mathfrak{l}+\mathfrak{u} .
$$

It follows that $\phi \in T_{\tilde{x}}^{*} \tilde{X}_{0}$ implies $l(\phi) \in T_{\tilde{x}}^{*} \tilde{X}$, as desired.

2.4 Lemma. $C h(\tilde{\mathcal{W}})=(K \times H) \cdot l(C h(\tilde{\mathcal{V}}))$. 
Proof. We begin the proof by observing that

$$
\tilde{Z}=(K \times H) \times{ }^{(K \cap L \times H)} \tilde{X}_{0} \text { and } \tilde{i}^{+} \tilde{\mathcal{W}}=\tilde{\mathcal{V}} .
$$

Hence we are in the situation of 2.1. Let $\tilde{Q}$ be a $(K \times H)$-orbit on $\tilde{Z}$ and let $\tilde{Q}^{\prime}=\tilde{Q} \cap \tilde{X}_{0}$. Choose a point $\tilde{x} \in \tilde{Q}^{\prime}$. Then it will suffice to check that

$$
l\left(\left(T_{\tilde{Q}^{\prime}}^{*} \tilde{X}_{0}\right)_{\tilde{x}}\right)=\left(T_{\tilde{Q}}^{*} \tilde{X}\right)_{\tilde{x}}
$$

Let $\phi \in\left(T_{\tilde{Q}^{\prime}}^{*} \tilde{X}_{0}\right)_{\tilde{x}}$. This means that $\phi \in \mathfrak{l}^{*}$ and that $\phi$ vanishes on $\mathfrak{k} \cap \mathfrak{l}+\mathfrak{b}_{x} \cap \mathfrak{l}$. Since both $\mathfrak{l}$ and $\mathfrak{u}+\theta \mathfrak{u}$ are $\theta$-stable, we deduce that $l(\phi)$ vanishes on $\mathfrak{k}+\mathfrak{b}_{x}$, or in other words that $l(\phi) \in\left(T_{\tilde{Q}}^{*} \tilde{X}\right)_{\tilde{x}}$.

Now it is not difficult to deduce the following result:

2.5 Lemma. Denote by $\beta_{0}$ and $\gamma_{0}$ the maps analogous to $\beta$ and $\gamma$, but defined with respect to $\mathfrak{l}$. Then

$$
\gamma \beta C h((\tilde{\mathcal{W}}))=\operatorname{Ad}(K) . l\left(\gamma_{0} \beta_{0} C h(\tilde{\mathcal{V}})\right)
$$

\section{A COMPUtation OF the ASSOCIATED VARIETy}

In this section we are going to compute $C h\left(j_{+} \mathcal{W}\right)$ for a $\mathcal{D}_{Z, \lambda}$-module $\mathcal{W}$ introduced in section 1. By applying the base change to the Cartesian square

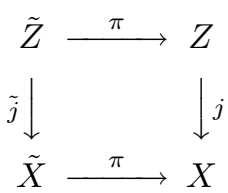

we obtain $\pi^{+}\left(j_{+} \mathcal{W}\right)=\tilde{j}_{+}\left(\pi^{+} \mathcal{W}\right)$. It follows now from 2.2 that it will suffice to compute $C h\left(\tilde{j}_{+} \tilde{\mathcal{W}}\right)$.

Our computation will be based on the formula for the characteristic variety of an open embedding from [Gi] (compare also [SV]). We briefly recall the formula. Let $j: U \longrightarrow X$ be an open embedding. Suppose that $f$ is a regular function on $X$ such that

$$
X \backslash U=f^{-1}(0) .
$$

For a $\mathbb{C}^{*}$-stable Lagrangian subvariety $\Lambda$ of $T^{*} U$ we set

$$
\Lambda^{\#}=\left\{\left(x, \xi_{x}+s \frac{d f(x)}{f(x)}, s\right):\left(x, \xi_{x}\right) \in \Lambda, s \in \mathbb{C}^{*}\right\} .
$$

Clearly $\Lambda^{\#}$ is a subvariety in $T^{*} X \times \mathbb{C}$.

3.1 Theorem. Let $\mathcal{F}$ be a regular holonomic module on $U$. Set $\Lambda=C h(\mathcal{F})$. Then

$$
C h\left(j_{+} \mathcal{F}\right)=C l\left(\Lambda^{\#}\right) \cap T^{*} X,
$$

where we have identified $T^{*} X$ with the subvariety $T^{*} X \times\{0\}$ of $T^{*} X \times \mathbb{C}$.

We remark that we can use either Zariski or analytic topology in computing the closure. This implies that $\Lambda^{\#}$ is closed in $T^{*} X \times \mathbb{C}^{*}$ (compare loc.cit., 2.1.14), and therefore

$$
C l\left(\Lambda^{\#}\right) \cap T^{*} X=C l\left(\Lambda^{\#}\right) \backslash \Lambda^{\#} .
$$


We return again to the setting of the flag variety and the problem of constructing a function $f$ on $\tilde{X}$ having the property

$$
f^{-1}(0)=\tilde{X} \backslash \tilde{Z} .
$$

But first we need a little more notation. As before we put

$$
\tilde{Y}=G / M U
$$

and denote by $\tilde{y}_{0}$ the identity coset. Then we have the natural map

$$
\pi_{0}: \tilde{Y} \longrightarrow Y
$$

determined by $\pi_{0}\left(\tilde{y}_{0}\right)=y_{0}$. Since $N \subset M U$, there exists a unique map

$$
\tilde{p}: \tilde{X} \longrightarrow \tilde{Y}
$$

such that $\tilde{p}\left(\tilde{x}_{0}\right)=\tilde{y}_{0}$. In order to construct a function $f$ with the above properties we shall adapt the method from [SW] to our setting.

Let $\langle$,$\rangle be a Killing form on \mathfrak{g}$. We define a bilinear form $F$ on $\mathfrak{g}$ by setting

$$
F(x, y)=\langle x, \theta y\rangle, \quad x, y \in \mathfrak{g} .
$$

Next we fix a basis $\left(X_{\alpha}, \alpha \in \Delta(\mathfrak{u})\right)$ and define a function $\phi$ on $G$ by the formula

$$
\phi(g)=\operatorname{det}\left[F\left(A d(g) X_{\alpha}, A d(g) X_{\beta}\right)_{\alpha, \beta}\right], \quad g \in G .
$$

Recall that $P$ normalizes $\mathfrak{u}$. A short computation yields

$$
\phi(g p)=(\operatorname{det}(A d(p) \mid \mathfrak{u}))^{2} \phi(g), p \in P .
$$

It follows that $\phi$ descends to $\tilde{Y}=G / M U$ and defines a regular function $\phi: \tilde{Y} \longrightarrow \mathbb{C}$. We will consider $\tilde{Y}$ endowed with $(G \times Z)$-action according to the formula

$$
(g, h) . g_{1} \tilde{y}_{0}=g g_{1} h^{-1} \tilde{y}_{0}, \quad g, g_{1} \in G, h \in Z .
$$

Evidently $\phi$ is $K$-invariant for the left $K$-action on $\tilde{Y}$. An important property of $\phi$ is given by the following lemma.

3.2 Lemma. $\phi(\tilde{y})=0$ if and only if $\tilde{y} \notin(K \times Z) . \tilde{y}_{0}$.

Proof. First of all, it is not difficult to show that $\phi$ is nonzero at any point of $(K \times Z) . \tilde{y}_{0}$. Suppose now that $y=A d(g) . y_{0}$ is a point in the boundary of $K . y_{0}$ and let $\tilde{y}$ be a point in $\tilde{Y}$ above $y$. Then $\phi(\tilde{y})=0$ if and only if $\left.\operatorname{det}\left[F\left(X_{\alpha}, X_{\beta}\right)\right]_{\alpha, \beta}\right]$ for $\alpha, \beta \in \Delta\left(\mathfrak{u}_{y}\right)$ is zero. We choose a $\theta$-stable Cartan subalgebra $\mathfrak{h}_{y}$ and the corresponding Levi decomposition $\mathfrak{p}_{y}=\mathfrak{l}_{y}+\mathfrak{u}_{y}$ as in 1.1. By the proof of 1.1, we can find a root $\alpha \in \Delta\left(\mathfrak{u}_{y}\right)$ such that $\theta \alpha \in \Delta\left(\mathfrak{p}_{y}\right)$. Therefore for any $\beta \in \Delta\left(\mathfrak{u}_{y}\right)$ we have

$$
F\left(X_{\beta}, X_{\alpha}\right)=\left\langle X_{\beta}, \theta X_{\alpha}\right\rangle=0 .
$$

This shows that $\left.\operatorname{det}\left[F\left(X_{\alpha}, X_{\beta}\right)\right]_{\alpha, \beta}\right]=0$, as claimed.

Finally we set $f=\phi \tilde{p}$. Now we can summarize the properties of the function $f$.

3.3 Lemma. (1) $f(\tilde{x})=0$ if and only if $\tilde{x} \notin(K \times H) L . \tilde{x}_{0}$.

(2) $f$ is $K$-invariant.

(3) $d f\left(k g \tilde{x}_{0}\right)=c A d(k) \rho(\mathfrak{u}), k \in K, g \in L$, and $c \in \mathbb{C}$. 
Proof. The first two properties are clear from the above discussion. To prove the third one we write $g=h g_{1}, h \in Z, g_{1} \in M$. Then we have

$$
f\left(k g \tilde{x}_{0}\right)=e^{4 \rho(\mathfrak{u})}(h) \phi\left(\tilde{y}_{0}\right) .
$$

A differentiation yields now the desired formula.

At this point we are ready to prove our main result. We draw attention to the notation introduced before 1.2. For a $(\mathfrak{g}, K)$-module $M$ we denote by $A S_{\mathfrak{g}}(M)$ the associated variety of $M$.

3.4 Theorem. Let $V_{\sigma}$ be an admissible $(\mathfrak{l}, K \cap L)$ - module with infinitesimal character determined by $\lambda \in \mathfrak{h}^{*}$ which is antidominant for $\mathfrak{g}$. Then the associated variety of the induced representation $W=\operatorname{Ind}_{\tilde{P}_{\mathbb{R}}}^{G_{\mathbb{R}}}\left(V_{\sigma} \otimes \mathbb{C}_{\rho(\mathfrak{u})}\right)$ is equal to

$$
A S_{\mathfrak{g}}(W)=C l\left(A d ( K ) \cdot ( l ( A S _ { \mathfrak { l } } ( V _ { \sigma } ) + \mathbb { C } ^ { * } \rho ( \mathfrak { u } ) ) ) \backslash A d ( K ) \cdot \left(l\left(A S_{\mathfrak{l}}\left(V_{\sigma}\right)+\mathbb{C}^{*} \rho(\mathfrak{u})\right) .\right.\right.
$$

Proof. First we recall the result of Borho and Brylinski [BB]. Let $\lambda \in \mathfrak{h}^{*}$ be antidominant. Then for a $\mathcal{D}_{X, \lambda}$-module $\mathcal{F}$ we have

$$
\gamma(C h(\mathcal{F}))=A S_{\mathfrak{g}}(\Gamma(X, \mathcal{F})) .
$$

We choose a $\mathcal{D}_{X_{0}, \lambda}$-module $\mathcal{V}$ such that $\Gamma\left(X_{0}, \mathcal{V}\right)=V$, and we realize $W$ as global sections of $j_{+} \mathcal{W}$ as in 1.2. Then by the discussion from the beginning of this section it suffices to determine $C h\left(\tilde{j}_{+} \tilde{\mathcal{W}}\right)$. We choose $f$ as in 3.3 and we apply 3.1. First we will identify $\Lambda^{\#}$. We extend $\beta(\gamma)$ to the map from $T^{*} \tilde{X} \times \mathbb{C}\left(\tilde{\mathfrak{g}}^{*} \times \mathbb{C}\right)$ to $\tilde{\mathfrak{g}}^{*} \times \mathbb{C}$ $\left(\mathfrak{g}^{*} \times \mathbb{C}\right)$, by making it act as identity on the $\mathbb{C}$-factor. Using 3.3.(3), we obtain

$$
\gamma \beta\left(\left\{\left(\tilde{x}, s \cdot \frac{d f(\tilde{x})}{f(\tilde{x})}, s\right): \tilde{x} \in \tilde{Z}, s \in \mathbb{C}^{*}\right\}\right)=\operatorname{Ad}(K) \cdot \mathbb{C}^{*} \rho(\mathfrak{u}) \times \mathbb{C}^{*} .
$$

If we combine this with 2.2, 2.5 and the result of Borho and Brylinski, we obtain

$$
\gamma \beta\left(\Lambda^{\#}\right)=\operatorname{Ad}(K) \cdot l\left(A S_{\mathfrak{l}}(V)+\mathbb{C}^{*} \rho(\mathfrak{u})\right) \times \mathbb{C}^{*} .
$$

Recall that $\beta$ is the quotient map for the $H$-action on $T^{*} \tilde{X}$. This implies that $\beta(C l(S))=C l(\beta(S))$, whenever $S \subset T^{*} \tilde{X}$ is $H$-stable. On the other hand, $\Lambda^{\#}$ is clearly $H$-stable and $\gamma$ is proper, so we obtain $\gamma \beta\left(C l\left(\Lambda^{\#}\right)\right)=C l\left(\gamma \beta\left(\Lambda^{\#}\right)\right)$. To conclude the proof of the theorem it suffices now to apply the formula $C l\left(\Lambda^{\#}\right) \cap$ $T^{*} X=C l\left(\Lambda^{\#}\right) \backslash \Lambda^{\#}$.

\section{REFERENCES}

[ABV] J. Adams, D. Barbasch, D. Vogan, The Langlands classification and irreducible representations for real reductive groups, Birkhäuser. MR 93j:22001

[B] D. Barbasch, Unipotent representations for real reductive groups, Proceedings of ICM 1990, Springer Verlag, 1991, pp. 769-777. MR 93m:22012

[BV] D. Barbasch and D. Vogan, Weyl Group Representations and Nilpotent Orbits, Representation Theory of Reductive Groups (P.C. Trombi, eds.), Birkhäuser-Boston, 1983, pp. 21-32. MR 85g:22025

[BB] W. Borho and J.-L. Brylinski, Differential operators on homogeneous spaces III, Invent. Math. 80 (1985), 1-68. MR 87i:22045

[Ch] J.-T. Chang, Remarks on localization and standard modules: the duality theorem on a generalized flag variety, Proc. of the Amer. Math. Soc. 117 (1993), 585-591. MR 93d:22016

[Gi] V. Ginsburg, Characteristic varieties and vanishing cycles, Invent. Math. 84 (1986), 327-402. MR 87j:32030 
[HMSW] H. Hecht, D. Miličić, W. Schmid, J. Wolf, Localization and standard modules for real semisimple groups I: The duality theorem, Invent. Math. 90 (1987), 297-332. MR 89e:22025

[Ka] M. Kashiwara, Systems of microdifferential equations, Progress in Math. 34, Birkhäuser, 1983. MR 86b:58113

[SV] W. Schmid and K. Vilonen, Characteristic cycles of constructible sheaves, Invent. Math. 124 (1996), 451-502. MR 96k:32016

[SW] W. Schmid and J. Wolf, A vanishing theorem for open orbits on complex flag manifolds, Proc. of the Amer. Math. Soc. 92 (1984), 461-464. MR 85i:32029

[Vo] D.Vogan, Representations of real reductive Lie groups, Progress in Math. 15, Birkhäuser, 1981. MR 83c:22022

Department of Mathematics, Cornell University, Ithaca, New York 14853

E-mail address: barbasch@math.cornell.edu

University of Zagreb, Geotechnical Faculty, 42000 Varaždin, Croatia

E-mail address: bozicevi@cromath.math.hr 